\title{
CUALIFICACIÓN Y SATISFACCIÓN LABORAL: UN ESTUDIO SOBRE LOS EMPLEOS PARA LOS QUE HEMOS SIDO FORMADOS PREVIAMENTE
}

\section{JOB QUALIFICATION AND SATISFACTION: A STUDY ABOUT EMPLOYMENT WHICH WE HAVE BEEN PREVIOUSLY TRAINED FOR}

Asenjo Fenoy, A., De Dios Chacón, M. \& Banqueri López, M. Universidad de Granada

Fecha de recepción: 28 de mayo de 2012

Fecha de aceptación: 11 de junio de 2012

Localizador: http:www.ugr.es/local/miguelgr/ReiDoCrea-Vol.1-Art.2-Fenoy-Dios-Banqueri.pdf

\section{RESUMEN}

Esta investigación, que ha contado con 80 sujetos, explora la relación entre las diferentes dimensiones de la satisfacción laboral y la cualificación del trabajo (relacionado o no con los currículos académicos previos). Se ha realizado utilizando Cuestionario de Satisfacción Laboral S20/23. Los resultados han sido significativos, confirmándose la hipótesis: hemos encontrado mayor satisfacción laboral en los sujetos que desarrollan trabajos cualificados y menor satisfacción en los que desarrollan trabajos no cualificados.

Palabras clave: cualificación, satisfacción laboral, formación previa

\begin{abstract}
This research study, which had 80 subjects, explores the relationship between different dimensions of job satisfaction and job qualification (related or not to prior academic curriculum). The study was carried out using Job Satisfaction Questionnaire S20/23. The results were significant, confirming the hypothesis: we found greater job satisfaction in subjects who carry out skilled jobs and lower satisfaction in those who carry out unskilled jobs.
\end{abstract}

Key words: qualification, job satisfaction, previous training 


\section{INTRODUCCIÓN}

En los últimos años se han publicado una gran cantidad de investigaciones sobre satisfacción laboral y otros aspectos relacionados con el trabajo. Así, encontramos por ejemplo investigaciones que estudian satisfacción y compromiso con la organización (Mathieu, 1991; Mathieu y Zajac, 1990), satisfacción, absentismo y la rotación del personal (Porteer, Steers, Mowday y Boulian, 1974) y satisfacción y rendimiento (Petty, McGee y Cavender, 1984) entre otros.

El interés por la satisfacción laboral reside en que es una dimensión que ocupa un lugar central en la consideración de la experiencia del hombre en el trabajo (Aldag y Brief, 1978). Se define como una respuesta emocional positiva al puesto de trabajo y que resulta de la evaluación de si el puesto cumple o permite cumplir los valores laborales de la persona (Locke, 1976, 1984). Por el contrario, la insatisfacción laboral sería la respuesta emocional negativa que ignora, frustra o niega los valores laborales del individuo (Locke, 1976). Esta insatisfacción, cuando es muy alta, se relaciona con una baja eficiencia organizacional, que puede expresarse además a través de falta de lealtad, negligencia, agresión o retiro debido a la frustración que siente el empleado (Flores, 1992).

Según Locke (1984) la satisfacción laboral está compuesta por varias dimensiones entre las que se encuentran las tareas a realizar, el salario, las posibilidades de promoción y la relación con los compañeros. Otros autores indican que también inciden factores no relacionados con la atmósfera laboral tales como la historia personal y profesional, la edad, el sexo, la formación, las aptitudes, la autoestima o el entorno cultural y socioeconómico (Shultz, 1990).

Una de las teorías más conocidos sobre satisfacción laboral es la formulada por Herzberg (1959) conocida como la teoría de los dos factores. Según ésta, existen dos grupos de factores que influyen en la satisfacción: los extrínsecos y los intrínsecos. Los primeros se refieren a las condiciones de trabajo en sentido amplio tales como salario, entorno o políticas de empresa. Según este modelo, los factores extrínsecos sólo pueden evitar la insatisfacción pero no pueden determinar la satisfacción, ya que ésta viene determinada por los factores intrínsecos tales como contenido, logro o responsabilidad. El modelo, por tanto, plantea que la satisfacción laboral sólo puede venir generada por los factores intrínsecos, a los que se llamó factores motivadores, mientras que la insatisfacción vendría determinada por factores extrínsecos, a los que se llamó factores higiénicos. Investigaciones posteriores comprobaron que la dicotomía entre ambos factores no está tan clara, sin embargo, se ha comprobado que la distinción entre factores intrínsecos y extrínsecos es importante en cuanto a que hay diferencias individuales respecto a la importancia que se le otorga a cada uno.

Sin olvidar que cada trabajador pueda otorgar más importancia a uno u otro factor, se ha comprobado que en general los principales factores que influyen en la satisfacción laboral son un trabajo intelectualmente estimulante, recompensas equitativas, condiciones favorables de trabajo y compañeros cooperadores. Así, los trabajadores prefieren puestos en los que puedan poner en práctica sus habilidades y que ofrezcan variedad de tareas, cierta libertad y retroalimentación sobre cómo lo están haciendo. Éstas características harían que el trabajo poseyese estímulos intelectuales. Hay que tener en cuenta además, que el nivel de retos ha de ser moderado ya que los que tienen pocos producen aburrimiento, y los que tienen demasiados, frustración. Por otro lado, los trabajadores quieren sistemas de pagos y políticas de ascensos que les parezcan justas. Cuando el salario está fundamentado en las exigencias del puesto, las habilidades del trabajador y el nivel de los sueldos del resto de trabajadores, el resultado tiende a ser la satisfacción. Del mismo modo ocurre con los ascensos que 
deben verse como honestos e imparciales. En cuanto al lugar de trabajo, los empleados prefieren entornos cómodos, seguros y limpios. Por último, es importante la presencia de compañeros con los que se establezca una amistad, ya que el respaldo de éstos se relaciona con el aumento de la satisfacción laboral (Robbins, 1998).

El objetivo de esta investigación, relacionada con estos últimos estudios ha sido comprobar si existen diferencias en los niveles de satisfacción laboral entre trabajos cualificados y no cualificados. La hipótesis es que los trabajos no cualificados tendrán menores niveles de satisfacción que los cualificados. Ésta hipótesis se basa en que los trabajos no cualificados (camareros/as, manipuladores de alimentos, secretariado, comerciales...) poseen por norma general características que suelen llevar a la insatisfacción del trabajador como las nombradas anteriormente: ausencia de recompensas equitativas, escasa variedad de tareas, poca libertad, o falta de retroalimentación positiva entre otras. Definiremos a continuación lo que entendemos por trabajo cualificado y no cualificado. Entendemos cualificado como cualquier trabajo relacionado con la preparación académica anterior del trabajador y como no cualificados aquellos en los que no haya una relación entre los estudios del trabajador y su desempeño en la empresa. Por último describimos nuestras variables la variable dependiente es el nivel de satisfacción y la variable independiente tiene dos dimensiones trabajo relacionado y trabajo no relacionado.

\section{MÉTODO}

\section{Participantes}

En esta investigación participaron 46 hombres y 34 mujeres (80 sujetos en total) de edades comprendidas entre los 20 y los 60 años $(M=30.43, D T=10.97)$, que fueron seleccionados al azar. De ellos, 41 sujetos desarrollaban trabajos cualificados, y los 39 restantes desarrollaban trabajos no cualificados.

\section{Instrumentos}

Se construyó un cuestionario que incluía las siguientes medidas:

- Datos sociodemográficos: edad, sexo y tipo de trabajo

- El Cuestionario de Satisfacción Laboral S20/23 de Meliá y Peiró (1998), compuesto por cinco dimensiones, las cuales miden Satisfacción con la Supervisión con un alfa de Cronbach de .95, Satisfacción con el Ambiente Físico con un alfa de .87, Satisfacción Intrínseca $(\alpha=.96)$, Satisfacción con las Prestaciones Recibidas con una consistencia interna de .79 y por último, la Satisfacción con la Participación con un alfa de .88. La consistencia interna para la escala total de Satisfacción Laboral fue igual a .96.

\section{Procedimiento}

Los participantes contestaron al cuestionario en un ambiente tranquilo, silencioso, con una temperatura agradable, y fuera de su lugar de trabajo. Se les dieron las siguientes instrucciones por escrito: "Habitualmente nuestro trabajo y los distintos aspectos del mismo nos producen satisfacción o insatisfacción en algún grado. Califique de acuerdo con las siguientes alternativas el grado de satisfacción o insatisfacción que le producen los distintos aspectos de su trabajo. Tal vez algún aspecto de la lista que le proponernos no corresponde exactamente a las características de su puesto de trabajo. En ese caso, entiéndalo haciendo referencia a aquellas características de su 
trabajo más semejantes a la propuesta, y califique en consecuencia la satisfacción o insatisfacción que le produce. En otros casos la característica que se le propone puede estar ausente en su trabajo, aunque muy bien podría estar presente en un puesto de trabajo como el suyo. Califique entonces el grado de satisfacción 0 insatisfacción que le produce su ausencia. Por ejemplo, si un aspecto que le propusiéramos fuera "residencias de verano", y en su empresa no le ofrecen tal cosa, califique entonces la satisfacción o insatisfacción que le produce no poder disponer de este servicio. Un tercer caso se le puede presentar cuando la característica que le propongamos no está presente, ni pueda estar presente en su trabajo. Son características que no tienen relación alguna, ni pueden darse en su caso concreto. Entonces escoja la alternativa, "4 Indiferente". Tal caso podría darse por ejemplo, si le propusiéramos para calificar "remuneración por kilometraje" y su trabajo, además de estar situado en su misma población, fuera completamente sedentario sin exigir jamás desplazamiento alguno. En todos los demás casos posibles escoja siempre para cada pregunta una de las siete alternativas de respuesta y márquela con una cruz".

Para la realización de los análisis estadísticos pertinentes se utilizó la versión 20.0 del programa estadístico "Statistical Package for Social Sciences" (SPSS 20.0).

\section{RESULTADOS}

A continuación, se describen las puntuaciones obtenidas en las principales escalas y medidas así como otras características de interés para el estudio.

Las puntuaciones medias y desviación típica en las diferentes dimensiones de la satisfacción en el trabajo aparecen en la Tabla 1.

Tabla 1. Puntuaciones medias y desviación típica en las dimensiones de la satisfacción en el trabajo

\begin{tabular}{|c|c|c|}
\hline & Media & Desviación Típica \\
\hline $\begin{array}{l}\text { SATISFACCIÓN } \\
\text { SUPERVISION }\end{array}$ & 4,71 & 1,70 \\
\hline $\begin{array}{l}\text { SATISFACCIÓN } \\
\text { AMBIENTE }\end{array}$ & 4,82 & 1,29 \\
\hline $\begin{array}{l}\text { SATISFACCIÓN } \\
\text { INTRINSECA }\end{array}$ & 4,19 & 2,02 \\
\hline $\begin{array}{l}\text { SATISFACCIÓN } \\
\text { PRESTACIONES }\end{array}$ & 3,85 & 1,42 \\
\hline $\begin{array}{l}\text { SATISFACCIÓN } \\
\text { PARTICIPACION }\end{array}$ & 4,22 & 1,64 \\
\hline
\end{tabular}

\section{Relación entre las dimensiones de la satisfacción laboral}

Para comprobar la relación entre las principales dimensiones de la satisfacción en el trabajo, se realizó un análisis de correlación de Pearson bivariadas. La matriz de correlaciones aparece en la Tabla 2. 
Tabla 2. Correlación entre las dimensiones de la satisfacción laboral

Supervisión Ambiente Intrínseca Prestaciones Participación

\begin{tabular}{|c|c|c|c|c|c|}
\hline Supervisión & -- & & & & \\
\hline Ambiente &, $75^{\star * *}$ & -- & & & \\
\hline Intrínseca &, $75^{* * *}$ &, $76^{\star \star \star}$ & -- & & \\
\hline Prestaciones &, $71^{* \star *}$ &, $64^{\star * \star}$ &, $72^{\star \star *}$ & -- & \\
\hline Participación &, $75^{* \star *}$ &, $69^{* \star \star}$ &, $70^{* \star *}$ &, $78^{* \star *}$ & -- \\
\hline
\end{tabular}

Como se puede observar en la Tabla 2 las diferentes dimensiones de la satisfacción laboral correlacionan positiva y significativamente en la muestra de nuestro estudio.

\section{Diferencias en la satisfacción laboral en función del tipo de trabajo (cualificado/no cualificado)}

Con el objetivo de comprobar las diferencias en la satisfacción en el trabajo en función del tipo de trabajo (cualificado/no cualificado) se realizó una prueba $t$ de student con cada una de las dimensiones de la satisfacción laboral (participación, prestaciones, supervisión, intrínseca y ambiente).

\section{$\square$ Cualificado $\square$ No cualificado}

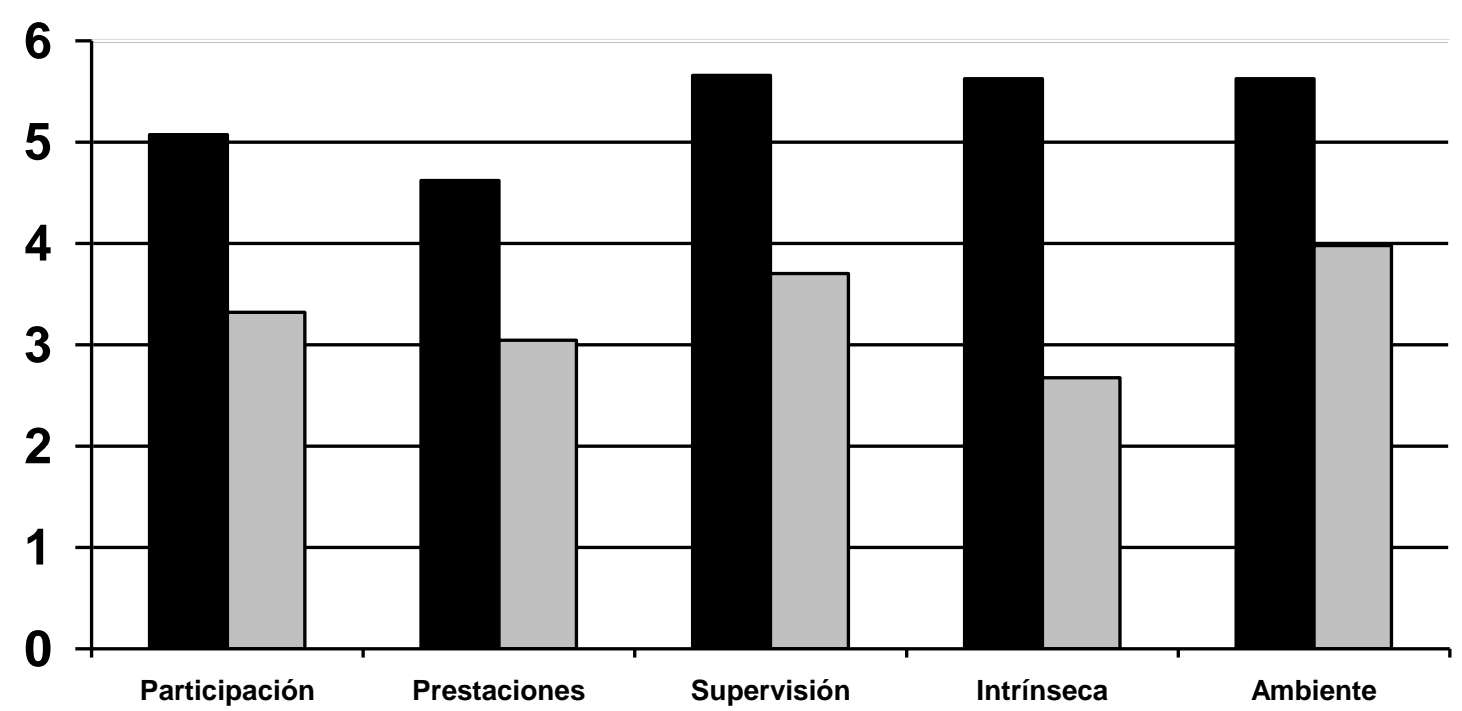

Gráfica 1. Puntuaciones medias en las dimensiones de la satisfacción en el trabajo en función del tipo de trabajo 
Los resultados mostraron diferencias significativas en todas las medidas (véase gráfica 1). De modo que el grupo cualificado presentó mayores niveles de satisfacción en todas las dimensiones. En la dimensión Satisfacción con la Participación $t(80)=5,60$, $p<.001$, el grupo cualificado obtuvo la puntuación $M=5,07$ y el no cualificado $M=3,32$. En la dimensión Satisfacción con las Prestaciones, $t(80)=5,91, p<.001$, el grupo calificado obtuvo una puntuación $M=4,62$ y el no cualificado $M=3,0449$. En la tercera dimensión, Satisfacción con la Supervisión, $t(80)=6,14, p<.001$, el grupo cualificado obtuvo la puntuación $M=5,66$ y el no cualificado $M=3,70$. En la cuarta dimensión, Satisfacción intrínseca, $t(80)=9,55, p<.001$, el grupo cualificado obtuvo $M=5,63$ y el no cualificado $M=2,67$. Por último, en la dimensión Satisfacción con el Ambiente, $t(80)=7,27, p<.001$, el grupo cualificado obtuvo $M=5,62$ y el no cualificado $M=3,98$. Como se puede observar vemos, existen diferencias estadísticamente significativas entre los dos grupos de nuestro estudio: el Cualificado y el No Cualificado en todas las dimensiones.

\section{DISCUSIÓN}

Nuestro estudio ha intentado explorar la relación entre la satisfacción laboral y el tipo de trabajo que se desempeñe. Los resultados han mostrado que existe una relación entre la satisfacción en los puestos de trabajo y si éstos están relacionados con la previa preparación de los empleados. De hecho el resultado general es que existe una diferencia significativa de satisfacción general entre los trabajadores cualificados y no cualificados $(t(80)=8,10, p<.001)$.

El cuestionario utilizado para medir satisfacción mide cinco dimensiones intrínsecas a la satisfacción; entorno físico, supervisión, intrínseca, prestaciones y participación. Existe una alta correlación entre las diferentes dimensiones (ver tabla 2). Esto es muy importante ya que nos muestra que el cuestionario seleccionado mide diferentes aspectos de la satisfacción y que niveles altos de satisfacción en una dimensión se relaciona con niveles altos de satisfacción en las demás dimensiones, obteniendo también una medida de satisfacción general.

Una vez sabemos que medimos satisfacción laboral y no otro constructo introducido como extraño, debemos hablar de que una variable que controlamos era el género de los participantes (46 hombres y 34 mujeres) aproximadamente la mitad de cada género. Los resultados no mostraron diferencias significativas entre la satisfacción que siente cada grupo, esto quiere decir que el sexo no parece influir en nuestra variable dependiente.

El objetivo principal del estudio consistió en comprobar si existen diferencias en la satisfacción laboral en función del tipo de trabajo (cualificado/no cualificado). Los resultados mostraron diferencias en todos los aspectos de la satisfacción. En el caso de la satisfacción con el entorno físico, las diferencias fueron estadísticamente significativas, de modo que, los trabajadores que más satisfechos están con las condiciones externas de su trabajo (iluminación, temperatura, higiene, limpieza, espacio, ventilación), son aquellos que tienen un trabajo cualificado, mientras que los que tienen un empleo que no requiere de sus conocimientos académicos no se encuentran satisfechos en esta dimensión. Una explicación podría ser que muchos de los trabajos no cualificados se realizan en entornos mal iluminados, ruidosos 0 calurosos (un supermercado, un bar, una cadena de montaje etc.)

Por otra parte, en la dimensión de la satisfacción con la supervisión también se obtuvieron diferencias estadísticamente significativas, lo que quiere decir que las 
personas con un trabajo cualificado están más contentas con la supervisión que reciben que las que no lo poseen. Una explicación sobre esto podría ser que las empresas con trabajos no cualificados suelen estar sujetas a planes de contratación temporales lo que hace que se considere siempre "nuevos" a los trabajadores por lo que éstos están continuamente sometidos a la presión de los supervisores.

En cuanto a la satisfacción intrínseca, es también estadísticamente significativa. Este dato era de esperar ya que los trabajos cualificados suelen permitir que los empleados utilicen una serie de habilidades que han aprendido y esto provoca cierta satisfacción ya que mejora el autoconcepto y las emociones son positivas hacia la tarea a realizar. En cambio en los trabajos no cualificados en empleado puede sentir que desperdicia sus capacidades, ya que suelen ser trabajos fáciles y repetitivos así como preminentemente físicos frente a los trabajos intelectuales más cualificados.

En cuarto lugar la dimensión de satisfacción sobre las prestaciones recibidas como puede ser el sueldo o los recursos de formación recibidos en la empresa, de nuevo se obtuvieron diferencias estadísticamente significativas. Este resultado puede entenderse desde la perspectiva de que la única prestación recibida en un trabajo no relacionado suelen ser el sueldo y éste suele ser bajo en contraposición a las horas que requiere. En cambio en los trabajos relacionados suele haber más alicientes que meramente el económico además de que éste suele ser más alto.

En último lugar, la satisfacción relacionada con la participación, también encontramos diferencias significativas entre ambos grupos. En los trabajos relacionados se suele permitir más la participación en toma de decisiones, sobre todo por el hecho de que existan tales tomas de decisiones, en cambio en los trabajos no relacionados ésta participación no existe, en primer lugar porque suelen existir férreas jerarquías que no permiten el paso de una línea a otra (un ejemplo sería que el dueño de un bar es el que decide qué comidas se sirven mientras que el cocinero sólo las prepara). La participación o más bien la posibilidad de participación en la empresa es un buen predictor de la satisfacción laboral.

En definitiva, hemos ratificado los estudios de Locke (1984), que afirmaba que la satisfacción es un compendio de dimensiones y que las descritas y muy probablemente algunas más como las descritas por este autor; tareas a realizar, clima laboral, etc.

Herzberg (1959) plantea un modelo por el cuál los factores extrínsecos pueden evitar la insatisfacción pero sólo los intrínsecos promueven la satisfacción. Probablemente hayamos corroborado esta visión ya que los trabajos no cualificados pueden tener valores extrínsecos positivos (el alto salario de un camionero), pero esto no hace que esté satisfecho si no que le salva de la total insatisfacción. Por ello los trabajadores encuestados con buen sueldo pero con trabajos no cualificados, no se encuentran satisfechos.

Por otro lado, creemos que habría que añadir en nuestro cuestionario ítems sobre las relaciones laborales en la empresa ya que se enfatizan en muchos estudios sobre satisfacción laboral como el de Robbins (1998).

\section{CONCLUSIONES}

Creemos que el objetivo con el que se inicia esta investigación ha sido cumplido. Ya que hemos encontrado que la cualificación en el trabajo influye en la satisfacción 
laboral. La satisfacción laboral ha sido ampliamente estudiada (Petty, McGee y Cavender, 1984). Este interés por el constructo estudiado en nuestra investigación, se explica porque está muy relacionado con la eficiencia en el puesto de trabajo, así como de la permanencia en la empresa, el absentismo, etc. Por lo tanto, es de vital importancia para que los trabajadores se encuentren a gusto y por ello rindan adecuadamente, no caigan en el absentismo y no abandonen la organización.

Por otro lado, nuestra investigación podría mejorarse de muy diversas maneras; un aumento en la muestra mejoraría la validez ecológica. La forma de elegir a los sujetos debería verse sometida a un análisis de muestra significativa al menos de la población española, esto no ha podida ser realizado debido a nuestra falta de recursos.

A su vez, la variable independiente podría ser descrita y medida con mayor atención, pues quizá existen trabajos relacionados con estudios previos que no son cualificados (formación profesional en peluquería) y que por lo tanto podrían dar lugar a resultados contradictorios.

Hemos controlado la variable extraña del género, pero podrían hacerse estudios controlando otras variables; como por ejemplo la edad. ¿Influye la edad en la satisfacción? Otras variables interesantes podrían ser las creencias religiosas quizá haya religiones con visiones sobre la vida laboral que podrían influir; si el trabajo es un castigo de Dios, quizá estaré más insatisfecho que si ésta es una bendición divina. Otros ejemplos de variables interesantes podrían ser el contexto más general. Quizá en épocas de crisis económicas estemos más o menos satisfechos con nuestro puesto de trabajo.

Estos son en general, las lagunas de nuestro estudio que podrían verse mejoradas en posteriores vías de investigación.

\section{BIBLIOGRAFÍA}

Aldag, R. J., Brief, A.P. (1978). Examination of alternative model of job satisfaction. Human relations, 31, 91-98.

Atalaya, M. (1999). Satisfacción laboral y productividad. Revista de psicología, III, 5, 46-6.

Flores, J. (1992). El Comportamiento Humano en las Organizaciones. Lima: Universidad del Pacífico.

Herzberg, F. (1959). The Motivation to Work. New York: John Wiley and Sons.

Meliá, J. L., Peiró, J. M. (1989). La medida de la satisfacción laboral en contextos organizacionales: El Cuestionario de Satisfacción S20/23. Psicologemas, 5, 59-74.

Locke, E.A. (1976). The nature and causes of job satisfaction: Handbook of Industrial and Organizational Psychology. Chicago: Rand McNally.

Locke, E.A. (1984). Job satisfaction. Social Psychology and Organizational Behaviour. Chichester: Wiley. 
Mathieu, J.E. (1991). A cross-level nonrecursive model of the antecedents of organizational commitment and satisfaction. Journal of Applied Psychology, 76(5), 607-618.

Mathieu, J.E. y Zajac, D.M. (1990). A review and meta-analysis of the antecedents, correlates, and consequences of organizational commitment. Psychological Bulletin, 108, 171-194.

Petty, M.M., McGee, G.W. y Cavender, J.W. (1984). A meta-analysis of the relationships bewteen individual job satisfaction and individual performance. Academy of Management Review, 9, 712-721.

Porter, L.W., Steers, R.M., Mowday, R.T. y Boulian, P.V. (1974). Organizational commiment, job satisfaction, and turnover among psychiatric technicians. Journal of Applied Psychology, 59, 603-609.

Robbins, S. (1998). Fundamentos del Comportamiento Organizacional. México: Prentice Hall.

Salgado, J.F., Iglesias, M., Remeseiro, C. (1996). Clima organizacional y satisfacción laboral en un pyme. Psicotgema, 8, 329-335.

Schultz, L. (1990). Social workers as expert witnesses in child abuse cases: A format. Journal of Independent Social Work, 1(5), 69-87. 